\title{
TRATAMENTO DA DOENÇA DE CHAGAS PELO NIFURTIMOX (BAYER 2502)
}

\author{
Alu iz io Prata ". Vanize Macêdo, : Gildẹte Porto * ", Iraceme \\ Sontos** " Jose A. Cerisola ** " o Noiron Silva ** **
}

Foram tratados 77 pacientes com doença de Chagas pelo nifurtimox, subdivididos em quatro grupos.

Grupo I, com 30 pacientes na fase aguda. Somente oito doentes usaram a droga durante 120 dias, na dose inicial de $15 \mathrm{mg} / \mathrm{kg}$ de peso corporal e posteriormente de $10 \mathrm{mg}$. Vinte e dois pacientes tomaram dose insuficiente. Em quatro doentes (50\%) o xenodiagnóstico tornou-se negativo pós-tratamento; destes, três fizeram reação de Machado Guerreiro, a qual estava negativa. Houve um óbito por insuficiência cardiaca no 50 dia de tratamento. Seis pacientes apresentaram polineuropatia periférica.

* Grupo II, com 15 pacientes na fase crônica, tratados em ambulatório. Oito abandonaram o tratamento, mas cinco em dez que tomaram placebo fizeram o mesmo. Dos seis que tomaram a droga durante 120 dias e fizeram pelo menos um xenodiagnóstico pós-tratamento, havia quatro que estavam com os exames negativos. $O$ tratamento não alterou o eletrocardiograma, a área cardíaca ou a radiografia do esôfago dos doentes e não evitou que um paciente viesse a desenvolver insuficiência cardíaca e um outro arritmia.

Grupo III, com 15 pacientes na fase crônica, tratados com $10 \mathrm{mg} / \mathrm{kg}$, durante 120 dias. Fizeram 12 xenodiagnósticos mensalmente, cada um com oito caixas contendo 10 $\mathrm{T}$. infestans após o tratamento e somente $28,5 \%$ dos pacientes apresentaram todos os exames negativos. Houve acentuada redução da parasitemia, mesmo nos doentes não curados. $O$ número de xenos positivos passou de $43 \%$ antes do tratamento para $24,4 \%$ após o mesmo e o número de caixas positivas caiu de $29,6 \%$ para $7 \%$.

Grupo IV, com 17 pacientes na fase crônica, tratados com $8 \mathrm{mg} / \mathrm{kg}$ ao dia, sendo que em oito durante 120 dias, em cinco durante 100 dias e em quatro durante 60 dias. $A$ percentagem de doentes com os 12 xenos negativos pós-tratamento foi de 56,25 . Também neste grupo houve redução de parasitemia, caindo a percentagem de xenos positivos de 76,5 antes do tratamento para 10,3 e a de caixas positivas de 28,6 para 3,1. Não há vantagem em se prolongar o uso do nifurtimox por mais de dois meses. As percentagens de cura são bem inferiores as obtidas na Argentina, Chile e Rio Grande do Sul.

$A$ droga produz muitas reações, sendo a mais importante a polineuropatia periférica.

Na terapêutica da doença de Chagas nenhuma droga conseguiu se impor de modo a ter ampla aplicação clínica ${ }^{11,20,22}$. Depois da evidência da ação tripanosomicida dos compostos nitrofuranos ${ }^{18}$, principalmente após suges- tão do uso de esquemas de duração prolongadá e devido ao interesse dos laboratórios Eaton, teve início uma série de ensaios clínicos com os compostos nitrofuranos (Furacin, NF'602 e NF 902).

\footnotetext{
* Universidade de Brasília

* * Universidade Federal da Bahia

** * Fundação Gonçalo Moniz

* * * Instituto de Diagnóstico e Investigação de la Enfermedad de Chagas Dr. Mario Fatala Chaben - Buenos Aires

* * * * Sanatório Espírita de Anápolis.

Recebido para publicacão em 20.6.1975
} 
No Brasil, embora doentes com fase aguda da doença de Chagas, após o tratamento com compostos nitrofuranos, tivessem xenodiagnósticos persistentemente negativos ${ }^{21}$ e em outros a reação de Machado Guerreiro se tomasse também negativa ${ }^{15}$ os resultados em geral foram mediocres. Porto e Cols ${ }^{19}$ trataram 11 doentes com a fase aguda, sendo três com Furacin na dose diária de $10-30 \mathrm{mg} / \mathrm{kg}$ de peso corporal, dois com Furacin na mesma dose associado à primaquina $(15 \mathrm{mg} / \mathrm{kg}$ ao dia), quatro com Furacin e NF $602(10-30 \mathrm{mg} / \mathrm{kg}$ ao dia) e dois com NF 602 e primaquina nas doses mencionadas, em esquemas que duraram de 21 a 266 dias de medicação. Somente um, dos nove doentes controlados, deixou de apresentar xenodiagnóstico positivo e chamou a atenção as reações de toxicidade, notadamente a polineuropatia $^{19}$. Na fase crônica da doença, a reação de Machado Guerreiro não se modifica e os resultados do xenodiagnóstico não são diferentes dos da fase aguda ${ }^{7}, 10$

Apresentaremos a seguir os resultados do ensaio clínico com um derivado do nitrofurfurilideno, conhecido como nifurtimox ou Bayer 2502, que é o 3-metil-4-(5-nitro furfurilidenoamino)-tetra-hidro-4H-I, 4-tiazina-1,1 dioxido. Além de ser uma nova substância, havia a recomendação dos fabricantes de que a dose diária do medicamento, para ser eficaz, deveria ser subdividida em três tomadas, o que até então não havia sido recomendado pelo fabricante dos outros nitrofuranos.

\section{MATERIAL E MÉTODOS}

Tratamos 77 pacientes com doença de Chagas pelo nifurtimox, fabricado pela casa Bayer. Dentre os doentes, 30 estavam na fase aguda da doença e 47 na crônica. Podemos dividi-los em quatro grupos, de acordo com a fase da doenç̧a e a maneira como foi usada a medicação e realizado o controle de cura.

Grupo I - No periodo de 1965 a 1972 tratamos 30 doentes internados no Hospital Prof. Edgard Santos, na Bahia, com fase aguda da doença de Chagas. Destes, 13 eram do sexo masculino e 17 do feminino e 5 eram brancos, 17 pardos e 8 pretos. A idade dos pacientes variou de 2 a 50 anos, sendo que em 18 era menor do que 9 anos. O diagnóstico foi estabelecido pelo encontro de $T$. cruzi no sangue periféricc. Dos 30 pacientes, 19 fizeram reação de Machado Guerreiro que foi reagente em 11 e não reagente em 8 . O Bayer 2502 foi dado em doses diárias de 8 a $25 \mathrm{mg} / \mathrm{kg}$, durante um a quatro meses, sendo ministrado em três tomadas. Somente oito doentes usaram o Bayer 2502 durante pelo menos 120 dias. O controle de cura foi realizado, durante o tratamento, pela pesquisa do parasito e, após o tratamento, pelo xenodiagnóstico com 10 ninfas do 49 ou 5 9 estágio de Rhodnius prolixus, sendo os triatomíneos examinados aos 30 e 60 dias. Quatro doentes não realizaram nenhum xenodiagnóstico pós-tratamento, 14 fizeram somente um e três outros somente dois xenos. Grupos de dois pacientes realizaram respectivamente, três, quatro, cinco e seis xenodiagnósticos. Somente um paciente completou os 10 exames programados. No controle de cura foram feitas reações de Machado Guerreiro em 23 doentes. Todos os pacientes permaneceram internados durante o tratamento. No período de seguimento continuaram internados ou ao abrigo da exposição à picada de triatomíneos infectados.

Grupo // - No período de 1968 a 1970 foram tratados 15 pacientes com doença de Chagas crônica, no Hospital Prof. Edgard Santos, no ambulatório da Clínica de Doenças Tropicais e Infectuosas da Faculdade de Medicina da Universidade Federal da Bahia. Quanto à forma clínica, havia quatro pacientes com a forma indeterminada, quatro com megas ou aperistalsis do esôfago, um com aumento da área cardíaca e sete com alterações eletrocardiográficas. Seis eram do sexo masculino e nove do feminino, havendo três brancos, oito pardos e quatro pretos, com idade entre 9 a 56 anos. Onze tinham xenodiagnóstico positivo e todos, exceto um, apresentaram reação de Machado Guerreiro positiva. Cada xenodiagnóstico foi feito com 10 ninfas do 40 ou $5 \%$ estágio de Rhodnius prolixus e lido após 30 e 60 dias. Os pacientes deveriam tomar o Bayer 2502 em doses variáveis de 5 a $15 \mathrm{mg}$ por quilo de peso corporal, subdivididas em três tomadas diárias, durante 90 a 120 dias. Como controle, concomitantemente, administramos placebo a 15 pacientes, que foram pareados com os tratados. No seguimento, três pacientes tratados não fizeram xenodiagnóstico. Este exame foi realizado, respectivamente, em número de uma, duas, quatro, seis, sete e nove vezes somente por um doente. $E$ em número de oito, dez e onze vezes, respectivamente, por dois pacientes. A reação de Machado Guerreiro foi realizada em onze doentes. Foram repetidos os eletrocardiogramas, as radiografias do coração e o exame radiológico do esôfago em dez pacientes. 0 
período de seguimento foi de 3 a 56 meses, sendo na grande maioria de um a dois anos. Os chagásicos que tomaram placebo foram submetidos a semelhante controle.

Grupo II/ - Em 1970 selecionamos 32 pacientes com xenodiagnóstico positivo e portadores da fase crônica da doença de Chagas, internados no Hospital Juliano Moreira, em Salvador. Os xenodiagnósticos foram feitos com ninfas do 40 ou 5 ọ estágio de Triatoma infestans, procedentes de Buenos Aires. Usou-se 80 ninfas em cada exame no 19 xeno, 40 no 2 ? 40 no 3 e e 20 ninfas no 4 ! xeno. As fezes dos dez triatomíneos contidos em cada caixa, eram examinadas, conjuntamente, 30,60 e 90 dias após terem sugado o sangue dos pacientes. Metade dos exames foi realizada em Salvador e metade em Buenos Aires. Dos 15 pacientes selecionados para tomar o Bayer 2502, oito tinham um xenodiagnóstico positivo, quatro tinham dois, dois tinham três e um tinha quatro, dos quatro xenos realizados em cada um. Sete eram do sexo masculino e oito do feminino. A idade variou entre 18 e 54 anos. Nenhum apresentava manifestações de insuficiência cardíaca. Todos eram esquizofrênicos.

Os pacientes tomaram o Bayer 2502 na dose diária de $10 \mathrm{mg}$ por quilograma de peso corporal, subdividida em três vezes, pela manhã, ao meio dia e à noite. A medicação foi dada por enfermeiros que assistiam à deglutição da droga. Terminado o tratamento fizemos um xenodiagnóstico mensal com 80 ninfas do 49 ou 5 ? estágio de $T$. infestans, durante um ano, como controle de cura e no final uma reação de Machado Guerreiro e um teste do latex com o reagente Chagas-latex, da Behringwerke. Em todo o perfodo os pacientes permaneceram internados no hospital.

Concomitantemente administramos placebo a 17 outros pacientes, vivendo nas mesmas condições, sendo seis masculinos e onze femininos e com idade entre 16 e 70 anos. 0 acompanhante foi o mesmo dos doentes tratadoos com o Bayer 2502 e se prolongou até 1972.

Grupo IV - Constituído de 17 pacientes c om doença de Chagas crônica, tratados e acompanhados no Sańatório Espírita de Anápolis, Goiás, no período de 1972 a 1974. Sete eram do sexo masculino e dez do feminino, sendo dez brancos, seis pardos e um preto. A idade variou entre 20 e 55 anos. Onze deles tinham sido internados no hospital psiquiátrico para tratamento, sendo cinco de oligofrenia, três de esquizofrenia e um, respectivamente, de epilepsia, estado paranoide climatérico e alcoolismo crônico. Os outros seis eram funcionários do hospital, em cujas residências não havia triatomíneos. Todos tinham reação de Machado Guerreiro positiva. Antes do trata. mento cada um deles foi submetido a quatro xenodiagnósticos no total de 28 caixas, com dez Triatoma infestans em cada uma. Dois exibiram somente um xenodiagnóstico positivo, dois tiveram dois, seis tiveram três e em sete todos os quatro xenodiagnósticos foram positivos (Tabela 3). Quanto ao número de caixas positivas, houve variações de 1 a 22, no total de 28 aplicadas em cada paciente. Os xenodiagnósticos foram examinados como os do grupo III.

O tratamento foi feito com o Bayer 2502 na dose diária de $8 \mathrm{mg}$ por quilo de peso corporal, subdividida em três vezes ao dia. A medicação foi tomada durante quatro meses por oito doentes, durante 100 dias por cinco e durante dois meses por quatro outros. Em doentes que não ficaram curados, após o 2 ọ xeno pós-tratamento a medicação foi ré-iniciada por mais dois meses em dois deles (Tabela 3, pacientes no 2 e 10) e por um mês em dois outros (Tabela 3 , pacientes no 13 e 14).

O controle de cura constou de xenodiagnósticos mensais, com 80 ninfas, durante 12 meses após o tratamento, conforme se procedeu para o grupo III. A única diferença foi a de que a metade das caixas foi examinada em Brasília ao invés de Salvador.

\section{RESULTADOS}

Grupo I - Dos oito doentes na fase aguda que tomaram o Bayer 2502, durante pelo menos 120 dias, na dose inicial de $15 \mathrm{mg} \mathrm{e}$ posteriormente de $10 \mathrm{mg}$ e que realizaram algum xeno de controle, havia três com reação de Machado Guerreiro negativa e de 2 a 6 xenodiagnósticos negativos e um com 3 xenodiagnósticos negativos mas sem realizar a reação de Machado Guerreiro. Nos outros quatro (50\%) o xenodiagnóstico se tornou positivo após o tratamento.

Independente do esquema de tratamento usado, 6 pacientes ficaram com Machado Guerreiro negativa, 17 estavam com a reação positiva e 7 não realizaram o exame. Quanto ao xeno, dos 26 que fizeram algum exame, 15 estavam positivos e 11 tinham todos os xenos negativos. Destes 11, havia 3 que realizaram um único exame, 2 doentes fizeram dois xenos, 2 outros três xenos e 1 doente, respectivamente, quatro, cinco, seis e dez exames. 
Quanto à tolerância, o tratamento foi suspenso, após oito dias, em um paciente com 5 anos de idade, que estava tomando $25 \mathrm{mg} / \mathrm{kg}$ e apresentou perda de equilíbrio. Um outro doente de 17 anos, faleceu com insuficiência cardfaca no 50 dia de tratamento com $15 \mathrm{mg} / \mathrm{kg}$. Pelo menos seis pacientes apresentaram polineuropatia sensitiva periférica e outros tiveram náuseas, vômitos, anorexia e diarréia.

Grupo // - Somente sete doentes completaram o esquema previsto e um deles não fez xenodiagnóstico após $\circ$ tratamento. Dos seis pacientes que completaram o tratamento $\mathbf{e}$ fizeram pelo menos um xenodiagnóstico de controle havia dois positivos e quatro negativos, sendo que destes o número de xenos era dois, sete, oito e nove, respectivamente. Em quatro deles foi feita a reação de Machado Guerreiro, após o tratamento, que continuou positiva.

Considerando-se todos os pacientes, os que completaram e os que suspenderam o tratamento, havia três com xenos positivos e nove negativos. Destes, havia um, respectivamente, com um, dois, quatro, sete, oito, nove e dez xenos e dois com onze xenos. Nos onze doentes que tomaram placebo e que tinham algum controle havia seis positivos e cinco negativos, sendo que dois fizeram um único xeno. As reações de Machado Guerreiro eram positivas em oito indivíduos e negativas em três, sendo que nestes havia dois que somente fizeram uma única reação e no outro uma reação positiva precedia as duas últimas negativas. Em oito pacientes que tomaram placebo a reação de Machado Guerreiro era positiva em seis e negativa em dois.

Durante o tratamento os pacientes apresentaram várias queixas, sendo mais freqüentes anorexia, perda de peso, insônia, nervosismo, extremidades frias, dormência nas extremidades e outras (Tabela 1). Também alguns pacientes que tomaram somente placebo apresentaram queixas semelhantes às dos que tomaram Bayer 2502 (Tabela 1). Houve dois doentes tratados e oito placebos que não apresentaram qualquer queixa.

Os oito doentes que abandonaram o tratamento parece que não o fizeram somente pelas reações apresentadas, pois dos que tomaram placebo também cinco tiveram semelhante comportamento.

Vale a pena mencionar que o tratamento não alterou o eletrocardiograma, a área cardfaca ou - megaesófago dos doentes e nem parece ter influido na evolução da doença, pois em um paciente surgiram arritmias e outro, quatro anos após, desenvolveu insuficiência cardfaca e ambos tiveram completado o tratamento pelo Bayer 2502.

Grupo III - Dos 15 individuos tratados 10 $(66,6 \%)$ estavam com xenodiagnóstico positivo após completar o controle de cura e $5(33,3 \%)$ apresentaram os exames negativos, sendo que destes somente 4 completaram os 12 exames de controle. Assim, a cura parasitológica ocorreu somente em $4(28,5 \%)$ pacientes. Ao mesmo tempo, unicamente $1(7,7 \%)$ dos 13 placebos continuava negativo (Tabela 2 ).

Embora poucos pacientes ficassem com xeno negativo, houve todavia uma redução da parasitemia pelo tratamento, pois antes do tratamento 26 dos 60 xenos (43\%) eram positivos e este número baixou para 34 em 139 (24,4\%) após o tratamento. Também houve redução no número de caixas positivas nos doentes tratados, passando de 78 em $263(29,6 \%)$ antes do tratamento a $78 \mathrm{em} 1112(7 \%)$ após o mesmo. Considerando-se somente os pacientes não curados, após o tratamento, o número de xenos positivo foi de $34 \mathrm{em} 88(38,6 \%)$ e o número de caixas positivas foi de 78 em $704(11,1 \%)$. Concomitantemente, nos pacientes após uso de placebo houve 87 xenos positivos em 162 realizados $(61,5 \%)$ e o número de caixas positivas foi de $299 \mathrm{em} 1104$ (27\%).

A reação de Machado Guerreiro, um ano após o tratamento, estava positiva em dez doentes e negativa em quatro dos pacientes tratados pelo Bayer 2502, sendo que dois destes tinham xenos negativos e dois positivos. Nos placebos a reação de Machado Guerreiro estava negativa em dois. $O$ teste do latex estava positivo em 14 doentes tratados pelo Bayer. 2502 e negativo em nenhum.

As manifestações de toxicidade foram semeIhantes às mencionadas no grupo II (Tabela 1).

Grupo IV - Durante o tratamento os xenos tornaram-se negativos em todos os pacientes. Após o mesmo somente $10 \mathrm{em} 17(58,8 \%)$ continuaram negativos (Tabela 3 ), mas excluindo-se o caso que fez somente um xeno de controle, tem-se 9 em 16 (56,25\%). Destes nove que apresentaram xenos negativos, dois haviam. tomado a droga durante quatro meses, quatro durante 100 dias e três a tomaram durante dois meses. Paradoxalmente, entre os sete positivos seis foram tratados durante quatro meses e somente um tomou a droga durante dois meses. Dos quatro retratados, em dois reapareceram xenodiagnósticos positivos, em um somente foram feitos dois xenodiagnósticos de controle, 


\section{TABELA 1}

Queixas dos pacientes após o uso do Bayer 2502 e de placebo, nos grupos II e III, em percentagens de doentes

Queixas dos pacientes

$$
\text { Grupo II }
$$

\begin{tabular}{|c|c|c|c|c|}
\hline 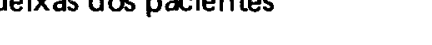 & Bayer 2502 & Placebo & Bayer 2502 & Placebo \\
\hline Anorexia & 30 & & 66 & 13 \\
\hline Perda de peso & 23 & & 80 & \\
\hline Tremores & & & 40 & \\
\hline Insônia & 23 & & 22 & \\
\hline Diarréia & & & 20 & 20 \\
\hline Dormência nas extremidades & 30 & 15 & 20 & \\
\hline Dores abdominais & 8 & & 13 & \\
\hline Astenia & 15 & & 7 & \\
\hline Nervosismo & 23 & 8 & & \\
\hline Extremidades frias & 23 & & & \\
\hline Cefaléia & 23 & 15 & & \\
\hline Artralgias & 8 & & & \\
\hline Vômitos & 8 & & 13 & \\
\hline Dor precordial & 8 & 8 & & \\
\hline Edema alérgico & 8 & & & \\
\hline Tonturas & 8 & 8 & & \\
\hline Mal estar & 8 & & & \\
\hline Lábios ressecados & 8 & & & \\
\hline Dispnéia & & 8 & & \\
\hline Arritmia & & 8 & & \\
\hline
\end{tabular}

Grupo III 


\section{TABELA 2}

Xenodiagnósticos em pacientes do grupo III após o tratamento com Bayer 2502 ou com placebo.

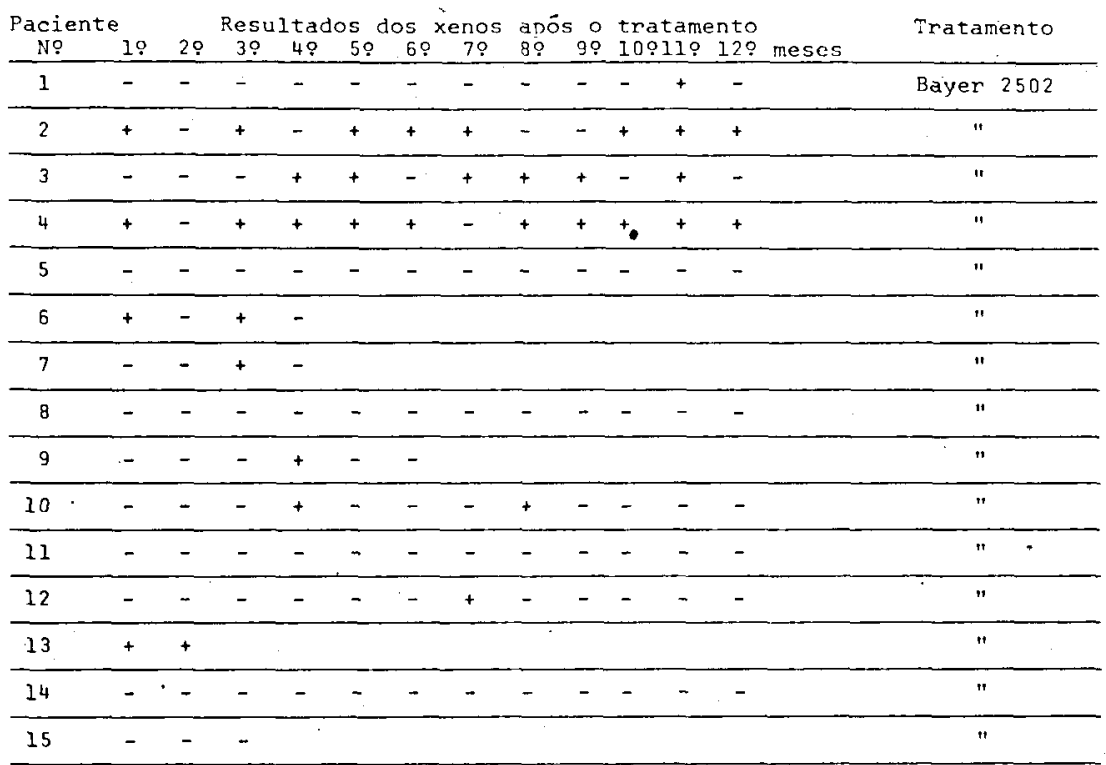

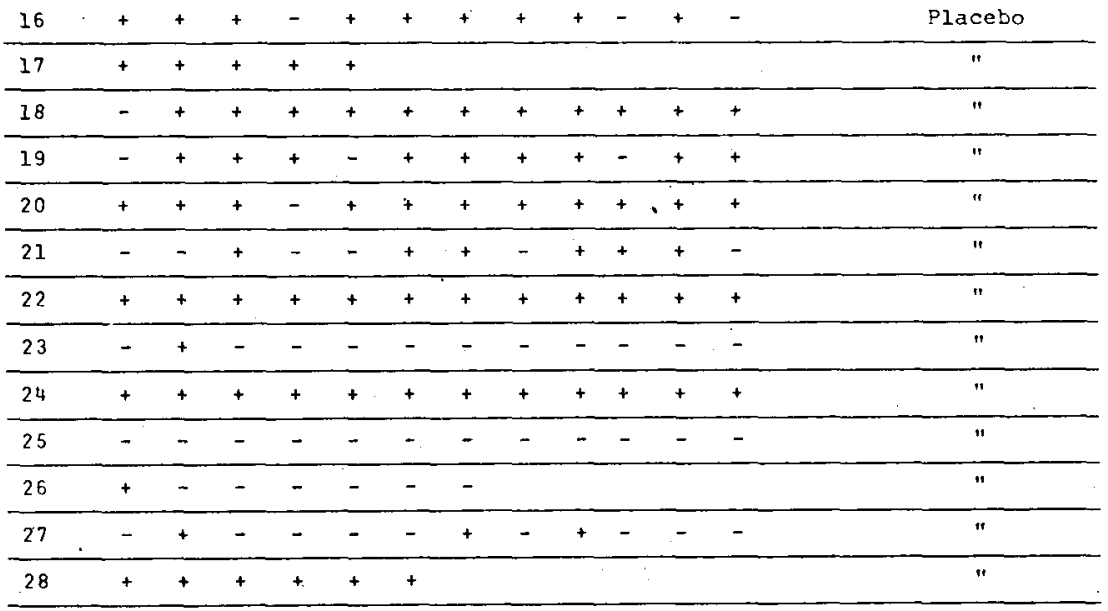




\section{TABELA 3}

Xenodiagnósticos em pacientes do grupo IV, antes, durante e após o tratamento com o Bayer 2502.

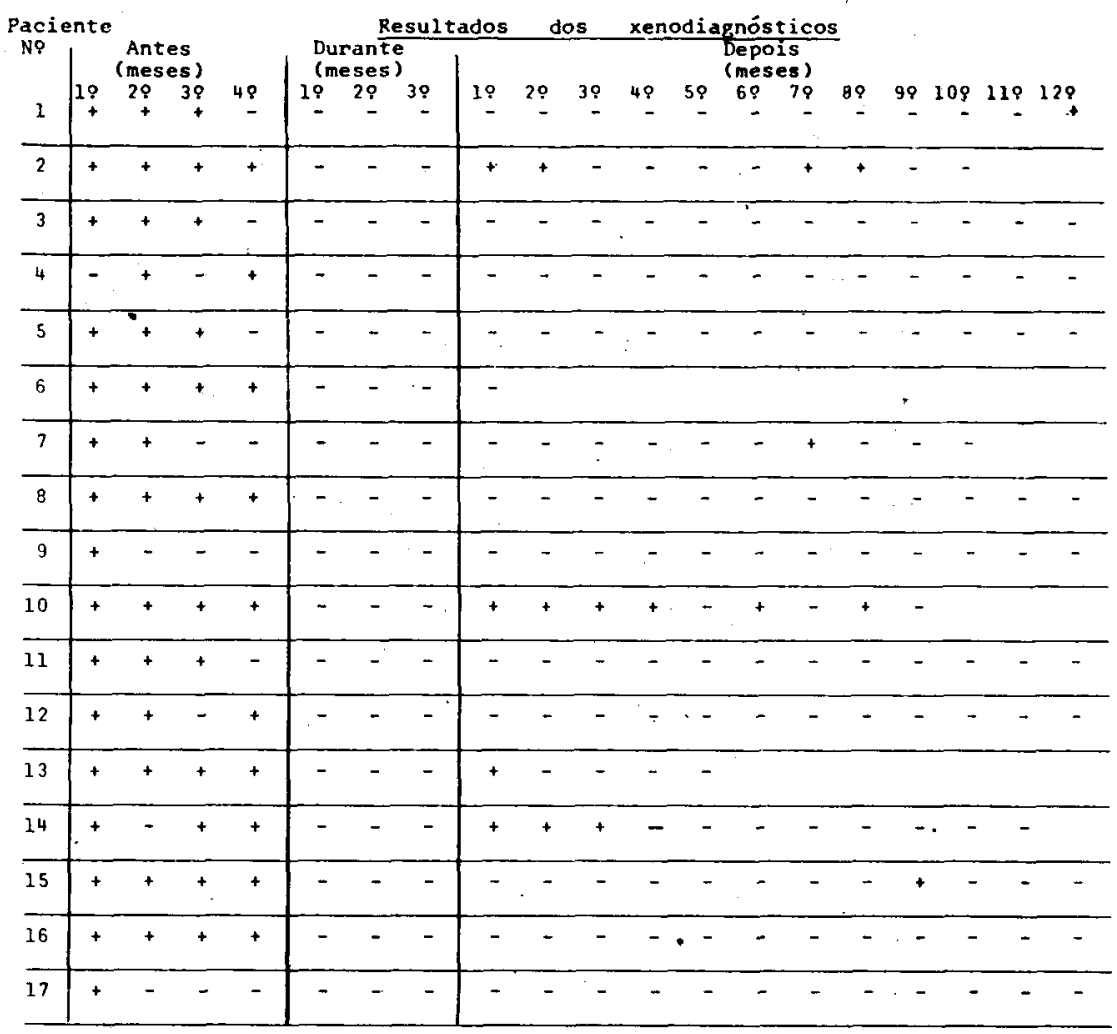

TABELA 4

Relação entre a eficácia do nifurtimox e o número de caixas positivas nos xenodiagnósticos antes do tratamento

\begin{tabular}{cc}
\hline $\begin{array}{c}\text { Xenodiagnóstico } \\
\text { positivo } \\
\text { No de caixas }\end{array}$ & $\begin{array}{c}\text { Pacientes que } \\
\text { continuaram com } \\
\text { parasitemia positiva }\end{array}$ \\
\hline$\%$ & $\%$ \\
$1-33$ & 53 \\
$34-66$ & 62,5 \\
$>66$ & 83 \\
\hline
\end{tabular}


ambos negativos e em outro o xeno continuou positivo durante $o$ tratamento.

Como no grupo III, também neste houve marcada redução na parasitemia. Antes do tratamento havia 52 xenodiagnósticos positivos em 68 feitos $(76,5 \%)$, com 136 caixas positivas das 476 aplicadas $(28,6 \%)$, enquanto que após o tratamento os xencdiagnósticos foram $18 \mathrm{em}$ $175(10,3 \%)$ e 44 caixas positivas em 1.400 $(3,1 \%)$. Mesmo considerando-se somente os exames dos pacientes não curados vemos que também neles houve redução da parasitemia, com 18 xenos positivos em 69 realizados (26\%) e com 44 caixas positivas em 552 (8\%). No final do perfodo de controle a reação de Machado Guerreiro estava positiva em oito pacientes, duvidosa em um, negativa em três e não foi realizada em quatro. Em três dos doentes com reação de fixação do complemento negativa havia dois com xenos também negativos.

As queixas dos pacientes foram as mesmas assinaladas na tabela 1, para os grupos anteriores. Vale a pena referir que um paciente teve várias crises convulsivas, aparentemente exacerbadas com o uso do medicamento. Exceto um pacíente que engordou um quilo, todos os outros emagreceram, respectivamente $3 \mathrm{~kg}$ (um doente), $4 \mathrm{~kg}$ (três doentes), $5 \mathrm{~kg}$ (cinco doentes), $6 \mathrm{~kg}$ (quatro doentes), $8 \mathrm{~kg}$ (dois doentes) e $9 \mathrm{~kg}$ (um doente). $O$ doente que engordou estava entre os que, após o tratamento, tiveram persistentes xenodiagnósticos negativos e também reação de Machado Guerreiro negativa.

Analisando em conjunto os resultados dos grupos III e IV, em função da parasitemia antes do tratamento, medida pelo número de xenodiagnósticos positivos, vemos que há certa correlação entre a elevada parasitemia e a persistência dos xenodiagnósticos positivos. Quando o número de xenodiagnósticos foi maior de $25-33 \%$ dos exames a percentagem de pacientes com xenodiagnósticos positivos foi de 44\%. De $50 \%$ para cima de xenos positivos no pré-tratamento correspondeu a $66 \%$ de pacientes que continuaram com os xenodiagnósticos positivos. Os números são mais significativos quando consideramos o número de caixas positivas nos xenodiagnósticos antes do tratamento (Tabela 4).

\section{COMENTÁRIOS}

Na fase aguda da doença de Chagas, da mesma maneira que a nitrofurazona ${ }^{15}$, temos a impressão que o nifurtimox pode melhorar a sintomatologia, embora, como afirmam outros $^{16,22}$, sem ser de modo espetacular. $O$ tratamento não evitou que uma nossa paciente falecesse no 50 dia do uso do Bayer 2502 devido à miocardite grave, ainda que o exame histopatológico mostrasse muitas formas intracelulares do parasito lesadas pelo medicamento. Do ponto de vista parasitológico, a parasitemia desapareceu durante o tratamento. Mesmo usando-se xenodiagnósticos somente com dez ninfas, repetidos poucas vezes, evidenciamos exames positivos em $\mathbf{5 0 \%}$ dos pacientes tratados. A reação de fixação de complemento estava negativa quando os xenos também o foram.

$\mathrm{Na}$ fase crônica da doença de Chagas o tratamento pelo Bayer 2502 não modificou as manifestações clínicas, eletrocardiográficas e radiológicas da doença, como se pode ver pelos resultados obtidos no grupo II. A percentagem de cura, quando se usou um controle rigoroso, foi de $\mathbf{2 8 , 5} \%$ dos doentes tratados na Bahia e $56,25 \%$ em Goiás. Mesmo nos doentes não curados houve redução da parasitemia, evidenciável por menor número de xenos positivos e, nestes, menos triatom(neos infectados. Basea dos em nossos resultados (grupo IV) achamos desnecessário prolongar o tratamento além de 60 dias, pois o uso da droga durante 120 dias não melhorou o percentual de cura. Embora tivéssemos retratados poucos doentes os resultados foram pouco influenciados pelo retratamento. Não conseguimos evidenciar alterações sorológicas após o tratamento, na fase crônica.

Tornou-se evidente a diferença dos resultados referidos em diferentes países. Os excepcionais resultados obtidos na Argentina e no Chile $9,23,24,25$ e também no Rio Grande do $\mathrm{Sul}^{26}$ não foram confirmados no Brasił, por nós e nem por Cançado e cols ${ }^{6}$ e nem em El Salvador, por Fernandez e cols ${ }^{13}$. Aliás, fato semelhante já havia sido observado com a nitrofurazona e derivados. Para explicar esta discrepância de resultados a hipótese mais provável é a diferença de raças de Trypanosoma cruzi.

$\mathrm{Na}$ fase aguda, quando se pode evidenciar facilmente a melhora da sintomatologia e a negatividade da parasitemia e da sorologia, 0 uso do nifurtimox dificilmente seria contestado. No entanto, devemos acentuar que, no momento, não temos evidências de que o tratamento pelo Bayer 2502 traz algum benefício para o doente, na fase crônica da doença. Experimentalmente, verificou-se a destruição 
das formas de $T$. cruzi $i^{1,3}$ mas os efeitos sobre a infecção crônica e quanto à prevenção de "megas" não foram bem evidentes ${ }^{3}$. Do ponto de vista clínico, as lesões existentes parecem não sofrer interferência em sua evolução. É importante saber se o tratamento, feito na forma indeterminada, previne o aparecimento de alterações eletrocardiográficas ou de perturbações nos diversos órgãos. A única transformação disponível é a de que o tratamento pode negativar a parasitemia em muitos doentes ou pelo menos reduzí-la, na grande maioria dos tratados. Talvez isto seja benéfico e por isso julgamos aconselhável continuar tratando os doentes. Se o medicamento produz a cura parasitológica somente com mais tempo poderemos responder. Vale a pena acentuar que quanto mais elevada é a parasitemia menor é a possibilidade dos xenos serem negativos após o tratamento. $\mathrm{O}$ ato de tratar o doente reduz o estigma que atemoriza muitos chagásicos, que é o de serem portadores de uma doença incurável.

Há necessidade de se conhecer melhor o comportamento da sorologia, na fase crônica, alguns anos após o tratamento. Talvez venta a suceder o mésmo que na sifilis, isto é, a reversão espontånea com a passagem dos anos, quando o tratamento for eficiente. No momento, a sorologia deve ser considerada como um bom método de controle de tratamento na fase aguda $^{8}$, mas não na crônica ${ }^{12,24,25}$.

Um problema quanto ao uso do Bayer 2502 é a sua tolerância, tendo a droga sido interrompida em muitos pacientes $3,5,6,7$, principalmente devido à polineuropatia. O Bayer 2502 para uns, como Marra ${ }^{17}$, não apresentaria vantagens sobre a nitrofurazona e a levofuraltadona, mas para outros, como Ferreira ${ }^{14}$, seria melhor tolerado. As crianças tolerariam melhor a droga 2,16 .

\section{SUMMARY}

Seventy seven patientes with Chagas disease were treated with nifurtimox. They were divided into 4 groups.

Group I consisted of 30 patients in the acute phase of the disease. Twenty two patients took an insufficient dose. Only 8 patients used the drug for 120 days in an initial daily dose of $15 \mathrm{mg} / \mathrm{kilogram}$ body weight for up to 2 weeks and later in a daily dose of $10 \mathrm{mg} / \mathrm{kilo}$ body weight. In 4 patients (50\%) the xenodiagnosis became negativa after treatment and in 3 the Machado Guerreiro test became negative. One patient died with acute cardiac failure on the 5th day of treatment. Six patients developed evidence of sensory peripheral neuritis while on treatment.

Group /I consisted of 15 outpatients in the chronic phase. They also took the drug for 120 days in a variable dosage of $5.15 \mathrm{mg} / \mathrm{kilo}$ body weight. They had at least one xenodiagnosis performed after treatment. In four patients xenodiagnosis became negative. The treatment had no effect on the electrocardiogram, degree of cardiomegaly, or appearance on barium swallow. Treatment did not prevent one patient from developing an arrhythmia and another cardiac failure.

Group /II also contained 15 patients in the chronic phase with positive xenodiagnosis treated with $10 \mathrm{mg} / \mathrm{kilo}$ body weight for 120 days. Twe/ve serial monthly xenodiagnosis were done after treatment using $80 \mathrm{~T}$. infestans (10 to a box) for each examination. In only $28.5 \%$ of the patients were all these xenodiagnoses negative. The frequency of parasitemia was decreased in the other patients but not eradicated. The number of xenodiagnoses positive before treatment was $43 \%$ and afterwards $24.4 \%$. The number of positive boxes diminished from $29.6 \%$ to $7 \%$.

Group IV consisted of 17 patients in the chronic phase with positive xenodiagnosis treated with $8 \mathrm{mg} / \mathrm{kg}$ per day. Eight were treated with this dose for 120 days, 5 for 100 days and 4 for 60 days. The percentage of patients with 12 serial xenodiagnoses negative after treatment was $56.25 \%$. A reduction in the incidence of positive xenodiagnoses from 76.5 to $10.3 \%$ also ocurred in this group and the number of positive boxes diminished from 28.6 to 3.1\%. There is no advantage in prolonging treatment beyond 2 months.

The percentage of cure is much less than that obtained in Argentina, Chile or Rio Grande do Sul (Brazill).

The drug produces many side effects especially a peripheral neuritis. 


\section{REFERÉNCIAS BIBLIOGRÁFICAS}

1. ANDRADE, Z.A. \& BRENER, Z. Ação da nitrofurazona (5-nitro-2-furaldeido-semicarbazona) sobre as formas intracelulares do Trypanosoma cruzi na doença de Chagas experimerital. Rev. Inst. Med. trop. de São Paulo. 11: 222-228, 1969.

2. BOCCA TOURRES, C.L. La enfermedad de Chagas en periodo agudo y su tratamiento con el Bay 2502. Bol. Chil. Parasitol. 24: 24-27, 1969.

3. BOCK, M.; GONNERT; R. \& HABERKORN, A. Studies with Bay 2502 on animals. Bol. Chil. Parasit. 24: 13-19, 1969.

4. BRENER, Z. Atividade terapêutica do 5-Nitro-2-Furaldeido-Semicarbazona (Nitrofurazona) em esquemas de duração prolongada na infecção experimental do camundongo pelo Trypanosoma cruzi. Rev. Inst. Med. trop. São Paulo. 3:43-49, 1961.

5. BUSTOS, A.G.; SOSA, E.V.; CONSTANZO, S.; WYBERT, L.A.; LUCIA, A. \& CARRIZO, F.A. Evolución clinica y de laboratório em niños y adolescentes con infección chagásica crónica tratados con Bay 2502 y con placebo. Bol. Chileno Parasit. 24: 63-65, 1969.

6. CANÇADO, J.R.; MARRA, U.L.; LOPES, M.; MOURÃ̃O, O.; FARIA, C.A.F.; ÁLVARES, J.M. \& SALGADO, A.A. Toxicidad $Y$ valor terapéutico del Bay 2502 en la enfermedad de Chagas crónica en tres esquemas posológicos. Bol. Chil. Parasit. 24: 28-32, 1969.

7. CANÇADO, J.R.; MARRA, U.D. \& BRENER, Z. Ensaio terapêutico clínico com a 5-nitro-2-furaldeido-semicarbazona (nitrofurazona) na forma cronica da doença de Chagas. Rev. Inst. Med. trop. S. Paulo. 6: 12-16, 1964.

8. CERISOLA, J.A. Evolución serologica de pacientes con enfermedad de Chagas aguda tratados con Bay 2502. Bol. Chil. Parasit. 24:54-59, 1969.

9. CHICERO, J.A.; SEGURA, E. \& QUATROCHI, J.C. Evolución clinico-parasitológica y tolerância a la droga de 33 niños con infección chagásica crónica tratados con Bay 2502. Bol. Chil. Parasit. 24: 59-62, 1969.
10. COURA, J.R.; FERREIRA, L.F. \& SILVA, J.R. Experiências con a nitrofurazona na fase crônica da doença de Chagas. $O$ Hospital, 62: 957-964, 1962.

11. COURA, J.R. \& SILVA, J.R. Aspectos atuais do tratamento da doença de Chagas. Arq. Bras. Med. $51: 283-290,1961$.

12. EJDEN, J. Efecto del Bay 2502 en adultos asintomáticos con infección chagásica crónica. Bol. Chil. Parasi. 24: 99-100, 1969.

13. FERNANDEZ, J.J.; CEDILLOS, R.A. \& GODOY, G.A. Tratamiento de la enfermedad de Chagas aguda con Bay 2502. Bol. Chil. Parasit. 24: 51-53, 1969.

14. FERREIRA, H.O. Comparacion de la tolerancia medicamentosa de la nitrofurazona, la levofuraltadona (NF-602) y el Bay 2502. Bol. Chil. Parasit. 24: 101-103, 1969.

15. FERREIRA, H.O.; PRATA, A. \& RASSI, A. Administração prolongada de nitrofurazona no tratamento da doença de Chagas aguda. O Hospital, 63: 1391-1396, 1963.

16. LUGONES, H.S.; PERALTA, F.; CANAL FEIJÓO, D. \& MARTELEUR, A.E. A Evolución de la sintomatologia clinica y la funcion hepatica en la enfermedad de Chagas aguda tratada con Bay 2502. Bol. Chil. Parasit. 24: 19-24, 1969.

17. MARRA, U.L. Comparación de la tolerancia medicamentosa de la nitrofurazona, levofuraltadona y el preparado Bay 2502. Bol. Chil. Parasit. 24: 38-42, 1969.

18. PACKCHANIAN. A. Chemotherapy of Experimental Chagas disease with Nitrofuran Compounds. Antibiotics and Chemotherapy, 7: 13-23, 1957.

19. PORTO, G.; PRATA, A. \& MACEDO, V. Dados não publicados.

20. PRATA, A. Estado atual da terapêutica especffica da doença de Chagas. Rev. Goiana Med. 9: (Supl.): 109-124, 1963.

21. PRATA, A. \& FERREIRA, H. Dois casos de doença de Chagas aguda aparentemente curados pela nitrofurazona. Gaz. Med. Bahia. 69: 25-29, 1969.

22. RASSI, A. \& FERREIRA,H.O. Tentativas de tratamento especifico da fase aguda da 
doença de Chagas com nitrofuranos em esquemas de duração prolongada. Rev. Soc. Bras. Med. Trop. 5: 235-262, 1971.

23. REBOSOLAN, J.B. Sensibilidad de los métodos de diagnóstico parasitológico en pacientes con enfermedad de Chagas aguda tratados con Bay 2502. Bol. Chil. Parasit.24: 49-50, 1969.

24. RUBIO, M. \& DONOSO, F. Enfermedad de Chagas en niños $y$ tratamiento con Bay 2502. Bol. Chil. Parasit. 24: 43-48, 1969.
25. SCHENONE, H.; CONCHA, L.; ARANDA, A.; ROJAS, A. \& ALFARO, E. Experiência terapeutica con el Bay 2502 en la infección chagásica crónica del adulto. Importancia del uso adecuado. del xenodianostico. Bol. Chil. Parasit. 24: 66-69, 1969.

26. SILVA, N.N.; KUHN, G.; SANTOS, J.F.C.; VON EYE, G. \& CHAHER, J.A.B. Eficacia e tolerancia do nitrofurfurilidene (1) na fase crônica da Molestia de Chagas. Rev. Soc. Bras. Med. Trop. 8: 325-334, 1974. 